\title{
PSORIATICARTHRITISANDSYSTEMICSCLEROSIS,ARAREASSOCIATION: A CASE REPORT
}

Gabriela Almeida Barbosa1,*, Robson Antônio Gonçalves ${ }^{1}$, Thyago Talles de Almeida Santana ${ }^{1}$, Sanna Paula Pires Mariano Campos ${ }^{1}$, Maria Roberta Melo Pereira Soares ${ }^{1}$, Alessandra de Sousa Braz ${ }^{1}$, Eutilia Andrade Medeiros Freire ${ }^{1}$

1.Universidade da Paraíba, João Pessoa (PB), Brazil.

*Corresponding author: gabrielaalmeidamed@gmail.com

\section{BACKGROUND}

Psoriasis is an inflammatory disease often associated with psoriatic arthritis (PA), represented by cutaneous-articular involvement. Systemic sclerosis (SS) is a disorder characterized by fibrosis and microvascular damage to the skin and internal organs. Fifteen reports are described in the literature that demonstrate the superposition of PA and SS and a common genetic and immunological basis has been suggested, without proof.

\section{CASE REPORT}

Male, 23 years, previously healthy, with a report of polyarthritis, diffuse skin rashes and FAN1:1280 homogeneous nuclear pattern, for 5 years, admitted to the Hospital das Clínicas (SP), for an unfinished diagnostic definition. He evolved with cough, weight loss, fever, with a diagnosis of pulmonary tuberculosis (TB) in 2015, with the completion of standard treatment. In 2016, he developed a TB miliary form and underwent new treatment. In 2017, he presented sepsis of pulmonary focus complicated by ischemic stroke due to septic embolism, evolving with generalized seizures, treated with phenobarbital and intercurred with toxic epidermal necrolysis, overcome after supportive therapy. In 2018, the patient restarted polyarthritis in the hands, wrists, knees and ankles, associated with the occurrence of dactylitis of the second right finger, nail dystrophy in the hands and feet (Fig. 1) and disseminated erythematousscaling lesions, with histopathology compatible with psoriasis. Laboratory tests showed mild anemia and an increase in VHS and PCR. He underwent a reassessment at the Hospital Universitário Lauro Wanderley (PB), presenting FAN 1:40 filamentary fibrillar, FR, antiphospholipid antibodies, anti-DNAn, anti-Sm, anti-Ro, anti-La, anti-Jo1, anti-RNP and ANCA negative. The patient filled CASPAR-2006 criteria for PA. During treatment, he experienced secondary failure to methotrexate and a new episode of TB. He started using leflunomide 20 mg/day, with partial response. Concomitantly, Raynaud's phenomenon (Fig. 1), digital ulcers (Fig. 2), skin thickening in the forearms, thighs, legs, hands and feet, in addition to radiographs of long hands and bones showing calcinosis (Fig. 3), fulfilling the ACR/EULAR-2013 criteria for ES.

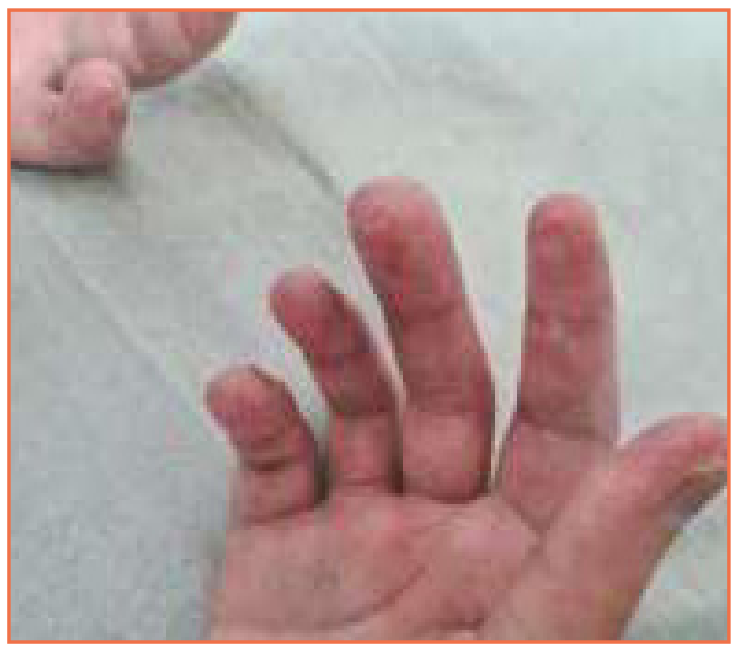

Figure 1. Left foot with Raynaud's phenomenon and dystrophy in the third finger.

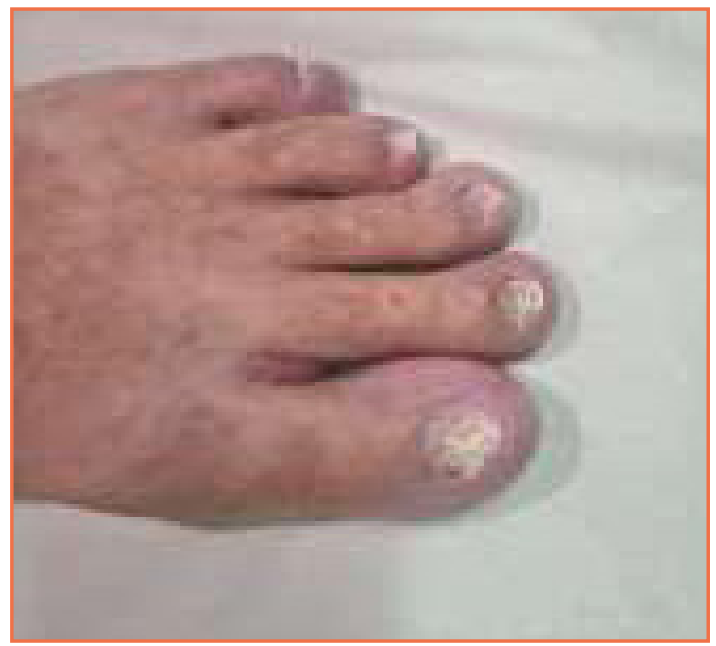

Figure 2. Right hand with digital nail ulcer. 

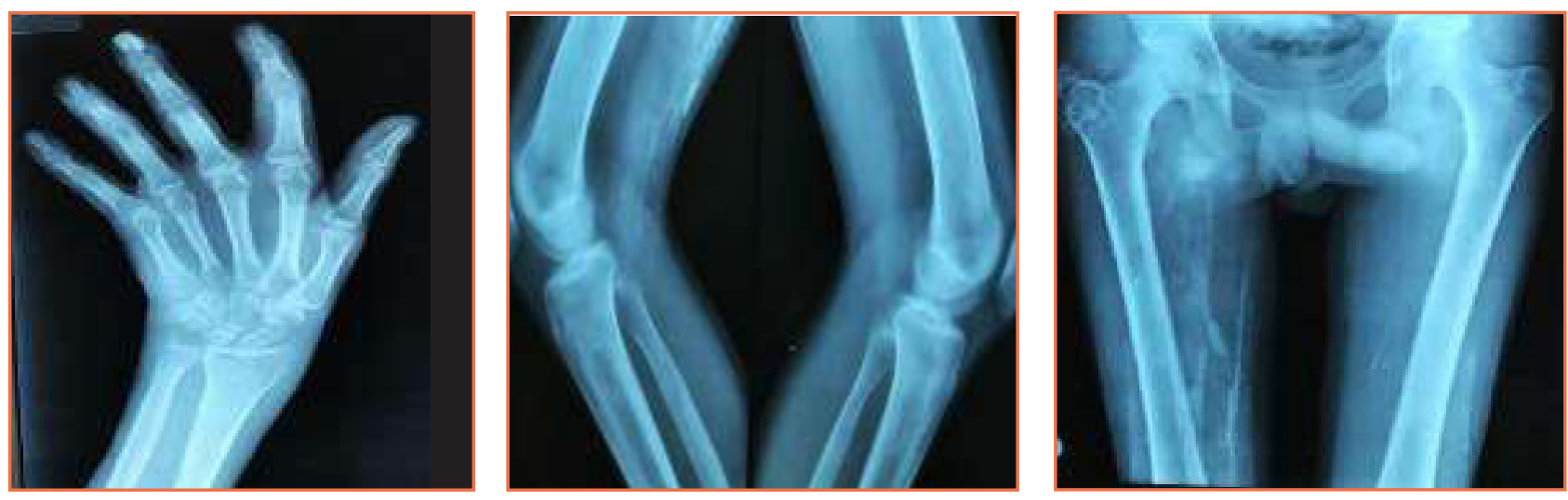

Figures 3. Radiography of D hand in PA, hip in PA and knees in profile, respectively, showing disseminated calcinosis.

\section{CONCLUSION}

Although the association of PA and SS is rare and little described, there is a suggestion that they have a common basis, as they are autoimmune, with involvement of the dermis, acro-osteolysis and periungual vascular abnormalities. In most cases in the literature and in the case reported above, PA preceded clinical manifestations and the diagnosis of SS, with the diffuse systemic form being the most commonly found. Such an association remains a challenge, with little known prognosis and clinical management. 